\title{
Optimisation of wave-power arrays without prescribed geometry over incident wave angle
}

\author{
Justin P. L. McGuinness, Gareth Thomas
}

\begin{abstract}
This paper describes the optimisation of arrays of wave energy converters (WECs) of point absorber type. The WECs are spherical in shape and operate in heave only. Previous work is extended to an optimisation of array layouts without a prescribed geometry. The objective function is chosen as the mean of the array interaction factor over a prescribed range of incident wave angles. This formulation forces the array to perform optimally over a specified range of wave angle, without direct concern for wavelength variations. Both constrained and unconstrained WEC motions are considered, with constrained optimisations limiting device displacements to two or three times the incident wave amplitude. The increased freedom in this more general optimisation results in a $70 \%$ to $140 \%$ increase in objective function values compared to the analogous linear array optimisations. As in previous studies of this nature, unconstrained arrays tend to contain closely spaced WECs and larger displacement amplitudes, whereas constrained optimal arrays are more widely spaced. It is shown that the prescribed range of incident wave angle has a great effect on the optimal array layout, with better performance achieved for smaller ranges of wave angle due to better tuning of the array members. A previously identified trade-off in linear arrays, between performance stability to different incident wave parameters, is shown not to apply to general array layouts.
\end{abstract}

Index Terms-Wave-Power, Arrays, Optimisation, Interaction, Point Absorber.

\section{INTRODUCTION}

The fundamental modelling on arrays of wave-power devices was presented independently by Evans [1] and Falnes [2]. Many subsequent papers have applied this theory to assess arrays of differing configurations, a relevant selection is given by [3]-[8]. More recently, optimisation of array layouts has become an important topic. General two-dimensional arrays of five devices were optimised by Fitzgerald [9] and reported in [6], where the array layouts were optimised by maximising the interaction factor in a point absorber regime. Arrays of heaving cylindrical wave energy converter (WECs) were similarly optimised using Genetic Algorithms by Child [7], within a full interaction regime. Optimisation of the mean performance of arrays of point absorbers are considered by McGuinness \& Thomas [10]-[13], where the arrays are optimised such that good performance is maintained even if optimal conditions are perturbed.

Manuscript received 16 March; accepted 27 March 2020; published $26 \mathrm{March}, 2021$. This is an open access article distributed under the terms of the Creative Commons Attribution 4.0 licence (CC BY https://creativecommons.org/licenses/by/4.0/). This article has been subject to single-blind peer review by a minimum of two reviewers. J.P.L. McGuinness received funding from a Government of Ireland Postgraduate Scholarship from the Irish Research Council and is gratefully acknowledged. J.P.L. McGuinness is with the Department of Mathematics, Cork Institute of Technology, Ireland. (email: j.mcguinness@cit.ie). G. Thomas is with the Department of Applied Mathematics, University College Cork, Ireland. (email:g.thomas@ucc.ie).
Within [3], [4], [6], [7], [9], arrays were optimised by directly maximising the performance of the array, either power absorbed or interaction factor, with respect to the array layout. This resulted in arrays which are high performing but also highly sensitive to small variations in array variables. This high sensitivity was identified and addressed in [8]. This was done by setting the objective function of the optimisation to be the mean of the interaction factor over a certain range of array variables, which resulted in arrays that performed well over a broader range of variables and thus were more stable to small changes in these variables.

One common problem with many of the above works is the large optimal motion amplitudes that result from unconstrained array optimisations. These large motions would cause severe control issues and also invalidate the underlying linear wave theory, which requires that the motions are small in some sense. Constrained optimisation of array layouts was investigated in [12], where the mean of the constrained performance of the arrays was maximised, with upper limits on WEC motions of two and three times the wave amplitude. It was found that there were some cases in which constructive interference could be stably maintained in the constrained regime. However this was achieved at a considerably reduced optimal performance compared to the unconstrained cases. It should also be noted that the optimal array layouts were altered by the application of constraints. In general, the inclusion of constrained motions resulted in more distributed arrangements when compared to unconstrained optimisations, with the removal of closely spaced groups of WECs for the most part. Overall, this work showed that the application of constrained motions resulted in a trade-off between optimal performance and stability of performance.

The majority of array optimisations involve prescribing an array geometry. The work presented in this paper extends previous studies to an array without a prescribed geometry, thereby allowing optimal arrays to attain any layout, subject to certain maximum and minimum device separations. The objective function of the optimisation is defined as the mean performance of the array over a prescribed range of incident wave angles. Unlike the objective functions used in [10][12], this is well defined regardless of the array layout. It is anticipated that this objective function will result in optimal arrays that are stable to changes in incident wave conditions.

The present approach does not include full interactions and follows the point-absorber implementation employed in [1], [3], [4], [8], [9]; the accuracy of this approximation is discussed in [5]. In this methodology, an external model is required to determine the device motions and for the chosen device geometry, the motions can be determined using the

Digital Object Identifier https://doi.org/10.36688/imej.4.1-10 
Havelock theory [14]. A similar optimisation method to that applied in [10]-[12] is utilised here, albeit with increased freedom and greater number of optimisation variables.

\section{MATHEMATICAL FORMULATION}

\section{A. Power Absorption Theory}

Consider an array of $N$ semi-submerged spherical point absorbers of radius $a$, which operate in heave only. It is assumed that linear wave theory is applicable and that regular long-crested waves of amplitude $A$, frequency $\omega$, wavelength $\lambda$ and wavenumber $k$ are incident on the array, in water of infinite depth with density $\rho$. The waves are incident at an angle $\beta$, which is measured in an anticlockwise direction from the positive $x$-axis. It has been shown by Evans [1] that the mean power absorbed by an array of $N$ WECs is given by

$P_{a b s}^{(N)}=\frac{1}{8} \mathbf{X}^{\dagger} \mathbb{B}^{-1} \mathbf{X}-\frac{1}{2}\left(\mathbf{U}-\frac{1}{2} \mathbb{B}^{-1} \mathbf{X}\right)^{\dagger} \mathbb{B}\left(\mathbf{U}-\frac{1}{2} \mathbb{B}^{-1} \mathbf{X}\right)$,

where $\mathbf{X}$ and $\mathbf{U}$ are complex time-independent column vectors of the exciting forces and velocities of the devices respectively, $\mathbb{B}$ is the radiation damping matrix and $\dagger$ denotes complex conjugate transpose. In this notation, the exciting force and velocity of body $m$ are given by $\operatorname{Re}\left[X_{m} e^{-i \omega t}\right]$ and $\operatorname{Re}\left[U_{m} e^{-i \omega t}\right]$ respectively. The power absorbed is maximised when

$$
\mathbf{U}_{\text {opt }}=\frac{1}{2} \mathbb{B}^{-1} \mathbf{X}
$$

which gives the maximum power absorbed by the array as

$$
P_{o p t}^{(N)}=\frac{1}{8} \mathbf{X}^{\dagger} \mathbb{B}^{-1} \mathbf{X}
$$

The device displacement amplitudes are given by the column vector $\xi=\operatorname{Re}\left[A \mathrm{D} e^{-i \omega t}\right]$, such that the column vector D represents the time-independent WEC displacements nondimensionalised with respect to the incident wave amplitude. These displacements can be related to the device velocities by

$$
\mathbf{U}=-i \omega A \mathbf{D} \text {. }
$$

The non-dimensional displacements are further separated into the WEC motion amplitudes and phases as

$$
D_{j}=\delta_{j} e^{i \psi_{j}},
$$

where $\delta_{j} \geq 0$ and $0 \leq \psi_{j}<2 \pi$.

There is no restriction placed on the geometry of the array, thus each array member has two variables that describe its position, with the exception of the first device with is fixed at the origin, without loss of generality. A cylindrical polar coordinate system $(r, \theta, z)$ is used to describe the position of each device, with the position of the $j^{\text {th }}$ device given by $(r, \theta, z)=\left(d_{j}, \alpha_{j}, 0\right)$, where $z=0$ corresponds to the water surface, $d_{j}$ is the distance from the origin to the $j^{\text {th }}$ device and $\alpha_{m}$ is the angle from the origin to the $m^{\text {th }}$ device measured positive in a counter-clockwise direction from the positive $x$ axis.

An absolute measure of the power absorption is not ideal as this does not give an indication of the power absorption capabilities of a chosen array compared to other arrays or to an isolated device. Thus the interaction factor is utilised which gives a measure of the relative performance of an array to the same number of isolated devices. For unconstrained motions, the interaction factor, or $q$-factor, is defined as

$$
q=\frac{P_{o p t}^{(N)}}{N P_{o p t}^{(1)}}
$$

where $P_{o p t}^{(1)}$ is the optimal power absorbed by a single isolated device. In the constrained regime, the averaged interaction factor $\bar{q}$ is defined as

$$
\bar{q}=\frac{P_{a b s}^{(N)}}{N P_{o p t}^{(1)}}
$$

\section{B. Point Absorber Approximation}

The point absorber theory assumes that the devices are sufficiently small so that they do not produce a scattered wave field. The corresponding mathematical assumption is that $k a \ll 1$. It has been shown in [5] that this approximation is valid for $k a \leq 0.8$. A value of $k a=0.4$ has been assumed by previous authors and is applied herein. Thus the far-field angular dependence of the WECs can be simplified, which allows the interaction factor to be expressed concisely as

$$
q=\frac{1}{N} \boldsymbol{\ell}^{\dagger} \rrbracket^{-1} \boldsymbol{\ell}
$$

while the analogous expression for the averaged interaction factor is

$\bar{q}=\frac{4 \pi(k a)^{2}}{N}\left(-\operatorname{Re}\left[(\mathcal{D}+i \mathcal{C}) \mathbf{D}^{\dagger} \ell\right]-\pi(k a)^{2}\left(\mathcal{C}^{2}+\mathcal{D}^{2}\right) \mathbf{D}^{\dagger} \rrbracket \mathbf{D}\right)$,

where $\ell$ is an $N$-component column vector with components $\ell_{m}=e^{i k d_{m} \cos \left(\beta-\alpha_{m}\right)}, \mathbb{J}$ is an $N \times N$ matrix with elements $\mathbb{J}_{m n}=J_{0}\left(k d_{m n}\right)$ for devices operating in heave and $J_{0}(x)$ is the zeroth order Bessel function of first kind. In this notation, $d_{n m}$ is the distance between the $n^{\text {th }}$ and $m^{\text {th }}$ devices. The constants $\mathcal{C}$ and $\mathcal{D}$ are called the Havelock coefficients. These are obtained as described in [14] and depend on the non-dimensional radius of the devices $k a$, which is fixed to $k a=0.4$ throughout this work. Therefore, $q$ is a function of the non-dimensional positions of the WECs (via $k d_{m}, k d_{m n}$ and $\alpha_{m}$ ) and of the incident wave angle $\beta$, while $\bar{q}$ is further dependent on the WEC displacements.

An important consistency relation was derived for the $q$ factor by [9] and reported in [6], where it was shown, within the point absorber regime for a single mode of motion, that

$$
\frac{1}{2 \pi} \int_{0}^{2 \pi} q(\beta) d \beta=1 \text {. }
$$

This is an important relation in this context, as it provides a limit on what can be achieved in terms of a wave angle optimisation of an array layout. It can also be shown that a symmetry in the interaction factor with respect to the incident wave angle exists and is

$$
q(\beta)=q(\beta+\pi)
$$




\section{Optimisation Method}

In the case of a non-prescribed geometry and a general 2D optimisation, the coordinates of all but the first device (which is fixed at the origin) are fed into the optimisation as variables; the non-dimensional coordinates utilised are $\left(k d_{j}, \alpha_{j}\right)$. The objective function for an unconstrained array is defined as

$$
I_{\beta}(k \mathbf{d}, \boldsymbol{\alpha})=\frac{1}{\beta_{u}-\beta_{l}} \int_{\beta_{l}}^{\beta_{u}} q(k \mathbf{d}, \boldsymbol{\alpha}, \beta) d \beta,
$$

where $k \mathbf{d}$ and $\boldsymbol{\alpha}$ are $N-1$ component vectors whose elements contain the $k d_{j}$ and $\alpha_{j}$ variables respectively. In an attempt to limit the search space of the optimisation and to restrict the array layout to a reasonable area, limits of $1 \leq k d_{j} \leq 20$ and $0 \leq \alpha_{j} \leq \pi$ are imposed for $j=2, \ldots, N$. These constrain the area of the array to within a semicircle in the upper half plane of radius 20, without loss of generality. To minimise calculation difficulties and to retain physical consistency, a further limit on the non-dimensional separation distance between any two WECs was also enforced such that $1 \leq k d_{n m} \leq 40$, which is consistent with the constraint on $k d_{j}$. A minimum separation of unity between any two WECs was enforced in order to avoid touching WECs and also to avoid numerical difficulties associated with small WEC separations.

A constrained objective function is also considered, where device motion amplitudes are limited to an upper value and the interaction factor $q$ replaced by the averaged interaction factor $\bar{q}$. Therefore, the constrained version of the objective function is given by

$$
\overline{I_{\beta}}(k \mathbf{d}, \boldsymbol{\alpha}, \boldsymbol{\delta}, \boldsymbol{\psi})=\frac{1}{\beta_{u}-\beta_{l}} \int_{\beta_{l}}^{\beta_{u}} \bar{q}(k \mathbf{d}, \boldsymbol{\alpha}, \boldsymbol{\delta}, \boldsymbol{\psi}, \beta) d \beta,
$$

where $\delta$ and $\psi$ are $N$-component vectors containing the displacement amplitude and phase variables respectively. For numerical implementation purposes, the displacement amplitude variable is reparameterised such that

$$
D_{j}=\widetilde{\delta}_{j} e^{i \psi_{j}},
$$

where $\widetilde{\delta_{j}}$ can take both positive or negative values and $0 \leq \psi_{j}<\pi$. It was found that the $\widetilde{\delta}_{j}$ variables were better behaved than the $\psi_{j}$ variables in terms of convergence of the optimisation. The displacements are implemented in this manner to improve the numerical optimisation, by increasing the range of $\delta_{j}$ and decreasing the range of $\psi_{j}$. Thus, the ranges of the displacement variables enforced are $-\delta_{\max } \leq \widetilde{\delta}_{j} \leq \delta_{\max }$ and $0 \leq \psi_{j} \leq \pi$.

There should be no need to examine different target values of incident wave angle, since it is clear that if an optimal array is found for one wave angle range, then a different target range should simply produce a rotation of the original optimal layout. Therefore, rather than examining different target wave angles, several range lengths are instead considered with the mid-point of each range remaining the same. For convenience, the target wave angle is assumed to be $\beta=\frac{\pi}{2}$ and the optimisation ranges are taken to have variations of $\pm \frac{\pi}{16}, \pm \frac{\pi}{8}$ and $\pm \frac{\pi}{4}$. Thus the corresponding optimisation ranges investigated are $\beta_{0} \in\left[\frac{7 \pi}{16}, \frac{9 \pi}{16}\right],\left[\frac{3 \pi}{8}, \frac{5 \pi}{8}\right]$ and $\left[\frac{\pi}{4}, \frac{3 \pi}{4}\right]$ which will be referred to as narrow, intermediate and broad-banded $\beta$ variation ranges respectively.

For unconstrained optimisations of this type, the optimisation contains $2(N-1)$ variables, corresponding to the radial and angular positions of each WEC when WEC 1 is fixed at the origin. The constrained optimisations contain an additional $2 N$ variables, comprising of the displacement amplitudes and phases of each WEC, resulting in a total of $4 N-2$ optimisation variables. In order to keep the optimisation feasible from a numerical perspective and to allow comparison with previous studies on linear arrays, an array of five WECs is examined in this paper. This results in eight optimisation variables for unconstrained motions and eighteen variables for the constrained optimisation.

Numerical calculations were performed on a Dell Latitude E6330 running Windows 7 with $8 \mathrm{~GB}$ of RAM and an Intel Core i3-3130M $(2.6 \mathrm{GHz})$ processor. The optimisation routine chosen to find the maximum of objective functions is NAG routine E04UCF (https://www.nag.co.uk/numeric/fl/manual/pdf/E04/e04ucf.pdf), with appropriate algorithms employed for the calculation of Bessel functions, matrix inversion and quadrature. This algorithm searches for the minimum value of the objective function using a sequential quadratic programming method. In order to find the maximum rather than the minimum, the negative of the objective function is supplied to the algorithm. A starting point is required as input to the optimisation algorithm. The algorithm initially uses the gradient of the objective function at this point to define a search direction, with the step size chosen such that a sufficient decrease in a Lagrangian type merit function is achieved. This procedure is repeated until an optimum is found. The algorithm E04UCF is essentially identical to the subroutine NPSOL as described by [15].

Optimisations of this type require an exhaustive search over the possible starting points of the variables, which results in a considerable demand on computational resources. For the unconstrained optimisation, all possible combinations of starting points with $k d_{j}=\{4,8,12,16,20\}$ and $\alpha_{j}=\left\{0, \frac{\pi}{5}, \ldots, \pi\right\}$ for $j=2, \ldots, 5$ were investigated, with combinations involving coincident devices forbidden. This involved a total of approximately 225,000 iterations in the optimisation, hence the large computation time of typically between five and ten hours for the unconstrained optimisation. The results of this scan of staring points were encouraging, as in each case the optimisation routine repeatedly converged to a single optimal solution, to an analogous one by symmetry or to one obtained by interchanging the WECs, indicating the stability of the optimisation and confirming the optimality of the solution.

The aspect of computation time is more severe for constrained motions, where an extra ten displacement variables must be scanned over. However, as discussed in [8], these displacement variables converged much better than the position variables, which allowed a more sparse scan of the stating point space to be implemented for these variables. Thus, the starting point space for constrained motions was $k d_{j}=\{5,10,15,20\}, \alpha_{j}=\left\{0, \frac{\pi}{4}, \ldots, \pi\right\}$ for $j=2, \ldots, 5$ and $\widetilde{\delta_{n}}=\{-3,0,3\}, \psi_{n}=\left\{0, \frac{\pi}{2}\right\}$ for $n=1, \ldots, 5$, with com- 
TABLE I

OPTIMAL POSITION VARIABLES FOR THE GENERAL 2D UNCONSTRAINED FIVE-DEVICE ARRAY

\begin{tabular}{c|cccc|cccc||c}
\hline$\left[\beta_{l}, \beta_{u}\right]$ & $k d_{2}$ & $k d_{3}$ & $k d_{4}$ & $k d_{5}$ & $\alpha_{2}$ & $\alpha_{3}$ & $\alpha_{4}$ & $\alpha_{5}$ & $I_{\beta}$ \\
\hline$\left[\frac{7 \pi}{16}, \frac{9 \pi}{16}\right]$ & 8.8663 & 4.2112 & 4.7345 & 9.2854 & 0.0840 & 0.0000 & 0.1911 & 0.1842 & 1.9451 \\
\hline$\left[\frac{3 \pi}{8}, \frac{5 \pi}{8}\right]$ & 8.8717 & 4.4099 & 8.4211 & 3.9625 & 2.9222 & 2.9272 & 3.0256 & 3.1416 & 1.7744 \\
\hline$\left[\frac{\pi}{4}, \frac{3 \pi}{4}\right]$ & 7.1035 & 3.4967 & 7.1750 & 3.6061 & 3.1416 & 3.1416 & 3.0018 & 2.8608 & 1.4466 \\
\hline
\end{tabular}

binations involving coincident WECs excluded. This resulted in approximately 185,000 calls of the optimisation routine, which is slightly less than the unconstrained optimisation. However, the optimisation had greater difficultly converging to the optimal set of variables and was required to make more steps within the minimisation process, thus making more calls to the objective function. This increased the computational effort significantly, resulting in run times of approximately 20 hours.

The optimisation results for constrained motions cannot be considered as reliable as those for unconstrained motions, as the optimisation routine did not converge repeatedly to the best set of variables found for an appropriate number of runs. Most cases resulted in the optimisation converging to the same optimal result approximately 100 times but in some cases the optimal solution was converged to only on three occasions. Thus the constrained optimisation is not as stable as the unconstrained version. Therefore, the results for the constrained arrays are not presented as optimal with confidence, but rather the best case found by the optimisation within a reasonable calculation time.

\section{Optimis ATION RESUlts}

This section presents the results of both the constrained and unconstrained $\beta$-optimisation of general two-dimensional five-WEC arrays. The best performing array variables and the resulting objective function values are listed in tables and the results discussed. It is worth noting that a value of $\alpha_{j}=0$ or $\alpha_{j}=\pi \approx 3.1416$ indicates that a WEC is placed on the $x$-axis, while a value of $\alpha_{j}=\frac{\pi}{2} \approx 1.5708$ would indicate that the device is placed on the $y$-axis. A detailed analysis of each optimal array is provided in Section IV.

\section{A. Unconstrained Motions}

The optimisation results for the unconstrained optimisation are shown in Table I for each of the ranges of incident wave angle considered. It is clear that better performance is achieved in the case of narrower wave angle ranges. This is to be expected, even when optimal motions are enforced for all wave angles, since the performance of an array with respect to $\beta$ is limited by (10). Thus, better performance can be achieved by contriving the array to perform well in a small $\beta$-range, since poor performance outside this range is of no concern. For broader $\beta$-ranges, (10) limits overall performance and results in lower objective function values.

As a consequence, the best performing array was for the narrow $\beta$-variation $\left(\beta \in\left[\frac{7 \pi}{16}, \frac{9 \pi}{16}\right]\right)$, where very high mean

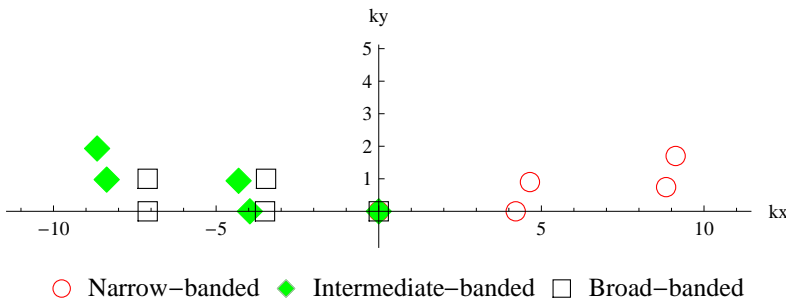

Fig. 1. Diagram of optimal unconstrained arrays for each range of incident wave angle

performance of $I_{\beta}=1.9451$ was achieved. The intermediate and broad wave angle ranges resulted in a mean performance drop of approximately $9 \%$ and $26 \%$ respectively relative to the narrow-banded case. As expected, the poorest performing array is for the broad-banded variation, where a mean performance of $I_{\beta}=1.4466$ was achieved.

A schematic showing the optimal array layouts for the unconstrained optimisation is presented in Fig. 1. It is clear that the optimal layouts are similar for each range of incident wave angle considered. In each case, the WECs are located close to the $x$-axis in two closely spaced pairs, where the lower limit of $k d_{n m}=1$ has been encountered. The narrow-banded optimal array is more spread out from the origin, whereas the broad-banded layout is closer to the origin. For the narrowbanded case, the WECs are positioned to the right of the origin, while the WECs are located to the left for the intermediateand broad-banded cases. This is probably due to the numerical optimisation arbitrarily converging to one orientation or the other, since the arrays will perform analogously if reflected about the $y$-axis via equation (11).

\section{B. Constrained Motions}

Two displacement amplitude limits are considered in the constrained optimisation, with the displacements limited to two or three times the incident wave amplitude. Tables II and III show the optimisation results for both constraints for all incident wave angle ranges considered. As a consequence of the large number of variables to be presented, the optimal WEC position variables are given in Table II, while the optimal displacement variables corresponding to the same arrays are displayed in Table III.

As with the unconstrained motions, the best performance is seen for the narrower ranges of $\beta$. The array with the highest optimal value of $\overline{I_{\beta}}=1.2137$ is the one optimised over the narrow-banded range of $\beta$ with the constraint $\delta_{\max }=3$. This indicates that, despite the motion constraint applied to 
TABLE II

OPTIMAL POSITION VARIABLES FOR THE GENERAL 2D CONSTRAINED FIVE-DEVICE ARRAY

\begin{tabular}{c|c|cccc|cccc||c}
\hline$\left[\beta_{l}, \beta_{u}\right]$ & $\delta_{\max }$ & $k d_{2}$ & $k d_{3}$ & $k d_{4}$ & $k d_{5}$ & $\alpha_{2}$ & $\alpha_{3}$ & $\alpha_{4}$ & $\alpha_{5}$ & $\overline{I_{\beta}}$ \\
\hline$\left[\frac{7 \pi}{16}, \frac{9 \pi}{16}\right]$ & 3 & 3.2631 & 2.9228 & 3.4293 & 1.8838 & 2.4752 & 2.9546 & 0.0000 & 0.9289 & 1.2137 \\
& 2 & 3.4037 & 2.9600 & 3.3490 & 1.9423 & 3.1416 & 0.2224 & 0.7160 & 2.1902 & 0.9293 \\
\hline$\left[\frac{3 \pi}{8}, \frac{5 \pi}{8}\right]$ & 3 & 5.2541 & 5.2541 & 2.5260 & 2.5260 & 1.2300 & 1.9116 & 0.1224 & 3.0192 & 1.0574 \\
& 2 & 5.3703 & 2.4178 & 5.3703 & 2.4178 & 1.8954 & 2.9652 & 1.2462 & 0.1764 & 0.8363 \\
\hline$\left[\frac{\pi}{4}, \frac{3 \pi}{4}\right]$ & 3 & 2.0031 & 2.3468 & 2.3468 & 2.0031 & 0.1996 & 0.9252 & 2.2164 & 2.9420 & 0.7085 \\
& 2 & 1.9465 & 2.4384 & 1.9465 & 5.1714 & 2.7676 & 1.5708 & 0.3740 & 1.5708 & 0.6350 \\
\hline
\end{tabular}

TABLE III

OPTIMAL DISPLACEMENT VARIABLES FOR THE CORRESPONDING GENERAL 2-D CONSTRAINED FIVE-DEVICE ARRAYS IN TABLE II

\begin{tabular}{c|c|ccccc|ccccc}
\hline$\left[\beta_{l}, \beta_{u}\right]$ & $\delta_{\max }$ & $\widetilde{\delta}_{1}$ & $\widetilde{\delta}_{2}$ & $\widetilde{\delta}_{3}$ & $\widetilde{\delta}_{4}$ & $\widetilde{\delta}_{5}$ & $\psi_{1}$ & $\psi_{2}$ & $\psi_{3}$ & $\psi_{4}$ & $\psi_{5}$ \\
\hline$\left[\frac{7 \pi}{16}, \frac{9 \pi}{16}\right]$ & 3 & -3.0000 & 3.0000 & -3.0000 & -3.0000 & -3.0000 & 1.7660 & 0.7140 & 2.1235 & 1.6480 & 3.1416 \\
& 2 & -2.0000 & -2.0000 & -2.0000 & 2.0000 & 2.0000 & 1.7927 & 1.6695 & 2.2991 & 0.8278 & 0.1598 \\
\hline$\left[\frac{3 \pi}{8}, \frac{5 \pi}{8}\right]$ & 3 & -3.0000 & -3.0000 & -3.0000 & -3.0000 & -3.0000 & 1.6092 & 0.4051 & 0.4051 & 1.9592 & 1.9592 \\
& 2 & -2.0000 & -2.0000 & -2.0000 & -2.0000 & -2.0000 & 1.6721 & 0.4837 & 2.0958 & 0.4837 & 2.0958 \\
\hline$\left[\frac{\pi}{4}, \frac{3 \pi}{4}\right]$ & 3 & -2.2652 & -3.0000 & 3.0000 & 3.0000 & -3.0000 & 1.3953 & 2.1598 & 0.3995 & 0.3995 & 2.1598 \\
& 2 & -2.0000 & -2.0000 & 2.0000 & -2.0000 & -2.0000 & 1.4044 & 2.4292 & 0.7421 & 2.4292 & 0.4403 \\
\hline
\end{tabular}

the WECs, constructive interaction is maintained. The same is true for the intermediate-banded optimal array with $\delta_{\max }=3$, where $\overline{I_{\beta}}=1.0574$ is achieved. Although this performance is lower, constructive interaction still dominates.

Significantly reduced performance is seen for the lower constraint of $\delta_{\max }=2$ in comparison to $\delta_{\max }=3$, as expected. In each case, the $\delta_{\max }=2$ arrays result in a reduction of between $10 \%$ to $25 \%$ compared with analogous $\delta_{\max }=3$ arrays. No array with this lower constraint achieved $\overline{I_{\beta}} \geq 1$, indicating that this constraint is more restrictive and destructive interference dominates. However, for the narrowbanded array, $\overline{I_{\beta}}=0.9293$ is achieved, which is only slightly lower than unity, indicating that modest performance is maintained.

In general, arrays optimised over larger ranges of $\beta$ return lower objective function values. For a small incident wave angle range, it is possible to tune the WECs to perform well for the conditions. However, for broader ranges, this is not possible as the same tuning cannot be done over all of this broader range of angles. Although equation (10) does not apply to constrained motions, the same argument can be made whereby the array cannot perform well at all angles, hence the lower performance for broader $\beta$ ranges.

For constrained motions, it does not appear that any of the WEC position variables reached either limit on $k d_{j}$ or $k d_{n m}$, indicating that no WEC is within close proximity to another. This is in line with the results of [12], where in general applying constraints on WEC motions resulted in more spread-out arrangements, with no grouped WECs.

It is clear from Table III that all WECs reached the maximum allowed amplitude constraint of $\delta_{\max }=2$ or $\delta_{\max }=3$ for each optimal array, with the exception of the broad-banded $\delta_{\max }=3$ array. This indicates that the WECs are working equally hard in each of these arrays and absorb the same amount of energy. In the narrow-banded optimal arrays, the WECs have different phases to each other. However, in the intermediate and broad-banded optimal arrays, at least one pair of WECs operate at the same phase. This is due to symmetry in the optimal layout, as will be considered in Section IV.

In the broad-banded $\delta_{\max }=3$ case, the motion amplitude of WEC 1 does not reach its maximum allowed value, unlike all other optimal constrained arrays. This indicates that reduced amplitude of this WEC somehow increases the mean performance with respect to $\beta$-variation. It may be that this reduces the peak performance slightly but improves the $\beta$-sensitivity of this optimal array, thus allowing the array to perform its best over the entire range of $\beta \in\left[\frac{\pi}{4}, \frac{3 \pi}{4}\right]$.

\section{Analysis of Optimal ArRays}

In this section, the optimal arrays found in Section III are analysed in more detail. For convenience, the analysis is conducted by the range of incident wave angle considered. Thus all constrained and unconstrained narrow-banded arrays are analysed in Section IV-A, similarly for intermediatebanded arrays in Section IV-B and broad-banded arrays in Section IV-C. In each case, a diagram of the optimal constrained and unconstrained array layouts is presented. The performance of each array with respect to $\beta$-variation and wavenumber variation is also assessed. Finally, the optimal WEC displacement amplitudes for the unconstrained array are presented and analysed.

\section{A. Narrow-Banded $\beta \in\left[\frac{7 \pi}{16}, \frac{9 \pi}{16}\right]$}

A diagram of the optimal constrained and unconstrained narrow-banded optimal arrays is shown in Fig. 2. It is clear that an appreciably different layout is found for each of the different displacement limits. The unconstrained array results 


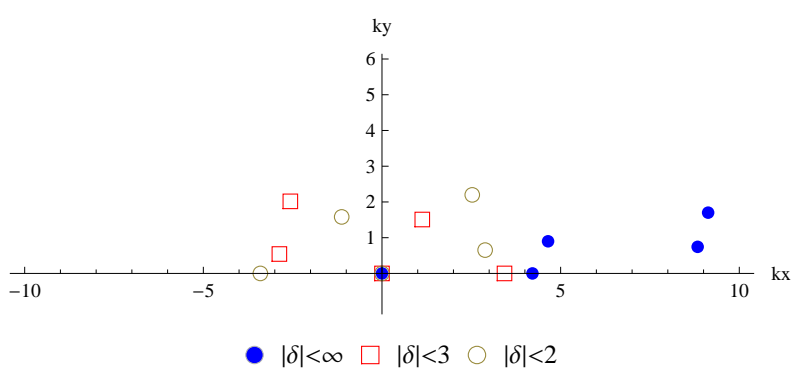

Fig. 2. Diagram of optimal arrays for narrow-banded range of incident wave angle $\beta \in\left[\frac{7 \pi}{16}, \frac{9 \pi}{16}\right]$

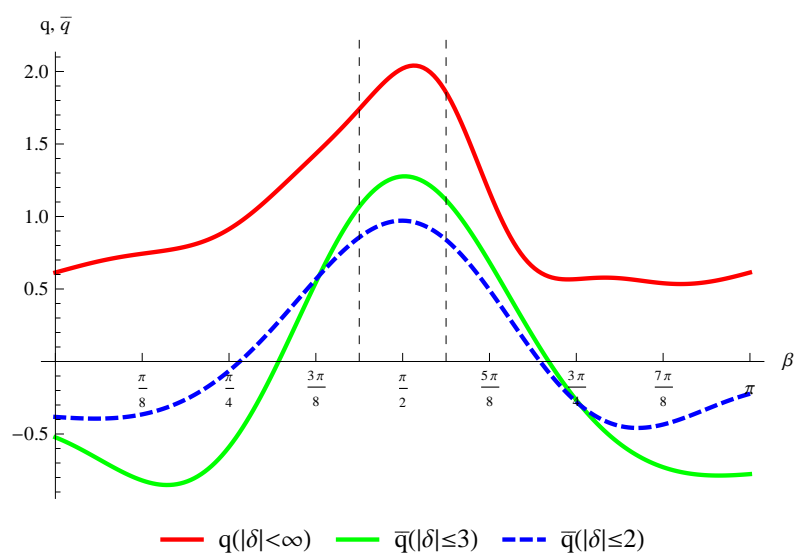

Fig. 3. Interaction factor against incident wave angle $\beta$ for optimal constrained and unconstrained five-WEC arrays, optimised for $\beta \in\left[\frac{7 \pi}{16}, \frac{9 \pi}{16}\right]$. The target range of wave angles is indicated by the vertical dashed lines.

in two pairs of closely-spaced WECs separated to the right of the origin. The separation between each of these pairs and the origin is approximately 4.5 units, while the paired WECs are placed as close to each other as allowed by the optimisation, with a unitary separation between them. The two constrained arrays are more central and contained within five units of the origin, with two WECs either side of the $y$-axis. Initially it would appear that the $\delta_{\max }=2$ and $\delta_{\max }=3$ constraints result in considerably different optimal layouts. However, an examination of both arrays shows that they are almost mirror images of each other about the $y$-axis. This suggests that the mirror arrangement of each array is analogously optimal for the given constraint but that the optimisation routine arbitrarily converged to the different symmetries in each case.

The performance of the optimal arrays with respect to $\beta$ variation is shown in Fig. 3. It is clear that each array is forced to perform best within the range of interest $\beta \in\left[\frac{7 \pi}{16}, \frac{9 \pi}{16}\right]$, since the performance of each array peaks in this range. The unconstrained array maintains a high interaction factor with $q \in[1.7,2.1]$ within the range of interest. The two constrained arrays show a considerably reduced performance and return averaged interaction factors of the order of half of the unconstrained case. However, constructive interference is maintained in the $\delta_{\max }=3$ case, with $\bar{q} \in[1.1,1.3]$ for all $\beta \in\left[\frac{7 \pi}{16}, \frac{9 \pi}{16}\right]$. Even though this is not the case for $\delta_{\max }=2$, adequate performance is maintained with $\bar{q} \in[0.85,1.0]$.

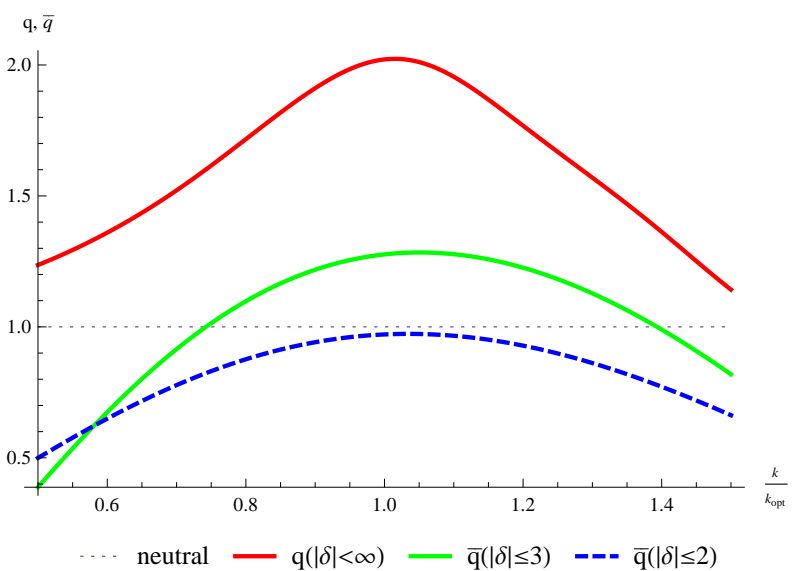

Fig. 4. Interaction factor against wavenumber $\frac{k}{k_{o p t}}$ for optimal narrowbanded constrained and unconstrained five-WEC arrays, with $\beta=\frac{\pi}{2}$.

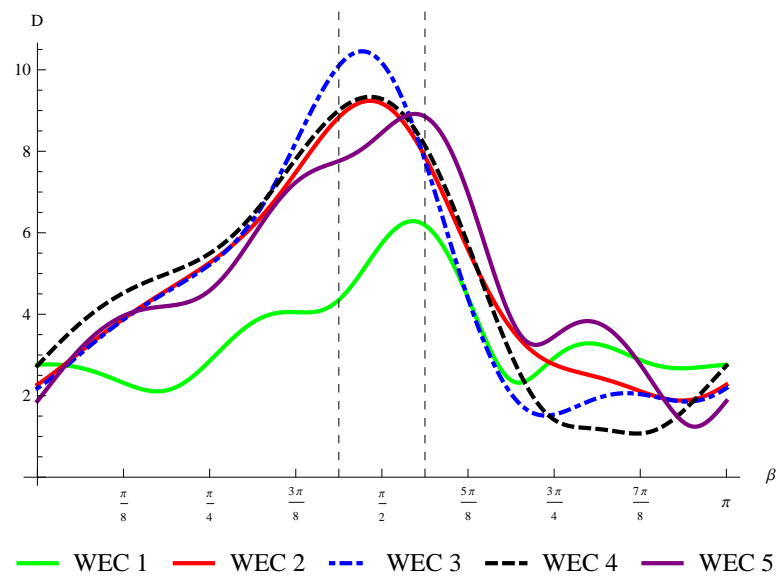

Fig. 5. Optimal displacement amplitudes against incident wave angle for optimal unconstrained five-WEC array, optimised for $\beta \in\left[\frac{7 \pi}{16}, \frac{9 \pi}{16}\right]$ (shown by vertical dashed lines)

The influence of wavenumber variation on each optimal narrow-banded array is shown in Fig. 4. The unconstrained optimal array maintains $q>1$ for up to a $50 \%$ variation about $k_{\text {opt }}$. The application of constraints causes a reduction in performance for the upper constraint, with constructive interference maintained for $\frac{k}{k_{o p t}} \in[0.75,1.4]$, indicating that at least a $25 \%$ change in $k$ is required to move from constructive to destructive interference. Due to the lower constraint having a greater effect on performance, $q<1$ holds for all values of $\frac{k}{k_{o p t}}$ considered. Each array is relatively stable overall to changes in wavenumber, with a small relative change in $k$ producing only a small change in $q$ or $\bar{q}$. It is noteworthy that the peaks in Fig. 4 occur at values slightly above $\frac{k}{k_{o p t}}=1$.

Finally, the predicted optimal WEC displacement amplitudes for the unconstrained array are shown in Fig. 5. It is clear that there is a correlation between the peak in performance in Fig. 3 and the peaks in WEC displacements in Fig. 5. The paired devices (WECs 2 - 5) exhibit larger motions than the relatively isolated WEC at the origin. However, all WECs have $\delta>4$ within the range of interest $\beta \in\left[\frac{7 \pi}{16}, \frac{9 \pi}{16}\right]$ and so violate 


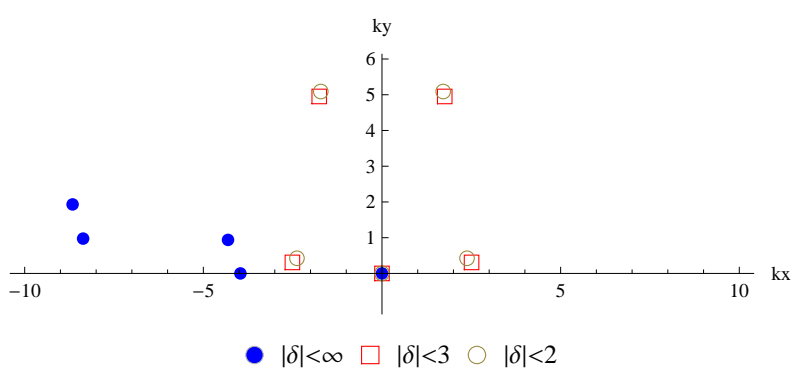

Fig. 6. Diagram of optimal arrays for intermediate-banded range of incident wave angle $\beta \in\left[\frac{3 \pi}{8}, \frac{5 \pi}{8}\right]$

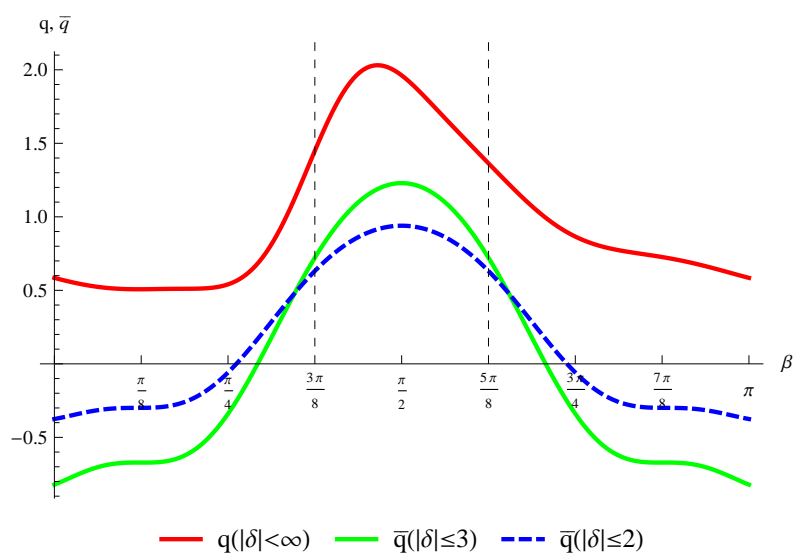

Fig. 7. Interaction factor against incident wave angle $\beta$ for optimal constrained and unconstrained five-WEC arrays, optimised for $\beta \in\left[\frac{3 \pi}{8}, \frac{5 \pi}{8}\right]$. The target range of wave angles is indicated by the vertical dashed lines.

the $\mathcal{O}(1)$ requirement of the model.

\section{B. Intermediate-Banded $\beta \in\left[\frac{3 \pi}{8}, \frac{5 \pi}{8}\right]$}

A schematic of the optimal array layouts for intermediatebanded $\beta$-ranges is shown in Fig. 6 for constrained and unconstrained motions. A similar optimal unconstrained layout to the narrow-banded case is found, with all WECs on the left of the origin rather than the right. Again, this contains two closely spaced pairs of WECs with unitary separation. The pairs have a separation of approximately four units between them and the isolated WEC at the origin. For both constrained arrays, an almost identical layout is found to be optimal, which is also symmetric about the $y$-axis.

The behaviour of the constrained and unconstrained optimal arrays to variation in incident wave angle is shown in Fig. 7. As with the narrow-banded case, the unconstrained array maintains constructive interference within the range of interest, with $q \in[1.35,2.05]$ for $\beta \in\left[\frac{3 \pi}{8}, \frac{5 \pi}{8}\right]$. The constrained arrays have reduced performance compared to the unconstrained case; in each constrained case destructive interference is present within the range of interest. The upper constraint resulted in $\bar{q} \in[0.75,1.25]$ for $\beta \in\left[\frac{3 \pi}{8}, \frac{5 \pi}{8}\right]$, whereas the lower constraint resulted in $\bar{q} \in[0.6,0.95]$. In contrast to the narrow-banded case, it is clear that a reduced performance occurs within the range of interest.

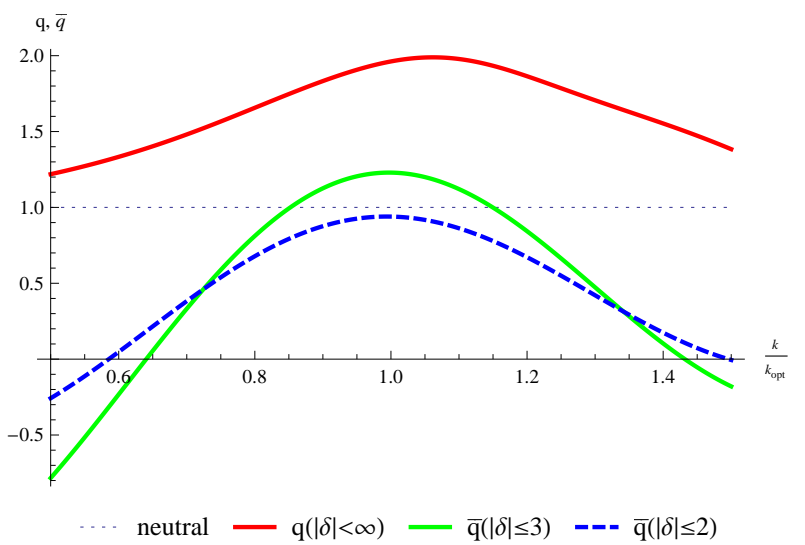

Fig. 8. Interaction factor against wavenumber $\frac{k}{k_{o p t}}$ for optimal constrained and unconstrained five-WEC arrays, optimised for $\beta \in\left[\frac{3 \pi}{8}, \frac{5 \pi}{8}\right]$, with $\beta=$ $\frac{\pi}{2}$.

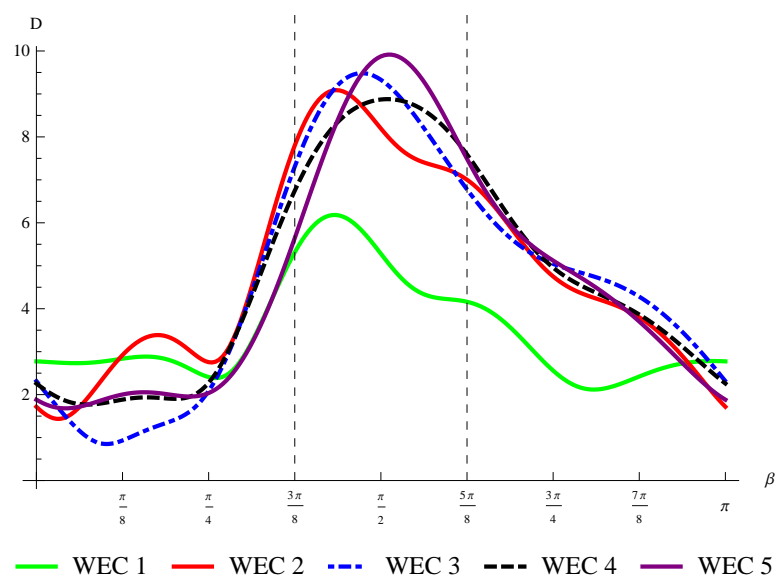

Fig. 9. Optimal displacement amplitudes against incident wave angle for optimal unconstrained five-WEC array, optimised for $\beta \in\left[\frac{3 \pi}{8}, \frac{5 \pi}{8}\right]$ (shown by vertical dashed lines)

The influence of wavenumber variations on the intermediate-banded optimal arrays is shown in Fig. 8. The unconstrained array maintains good performance for up to a $50 \%$ change in $k$, with $q>1.2$ for all values considered. Note that the peak in performance is achieved at $\frac{k}{k_{o p t}} \approx 1.06$, not $\frac{k}{k_{o p t}}=1$ as expected. A reduced performance and an increased sensitivity is seen for the constrained arrays. The lower constraint possesses destructive interference for all wavenumbers considered, with a $30 \%$ change in $k$ resulting in a $50 \%$ drop in $\bar{q}$. Constructive interference with $\bar{q}>1$ is seen for the upper constraint for $\frac{k}{k_{o p t}} \in[0.85,1.15]$. This indicates that a $15 \%$ change in wavenumber results in a destructive interference. Note also that a $40 \%$ change in $k$ for the upper constraint results in negative $\bar{q}$ values, indicating that the array is injecting power into the water rather than absorbing it.

The displacement amplitudes for the optimal unconstrained intermediate-banded array are presented in Fig. 9. This once more shows that the WEC displacements are largest for range of interest and so have a strong correlation with the high $q$ - 


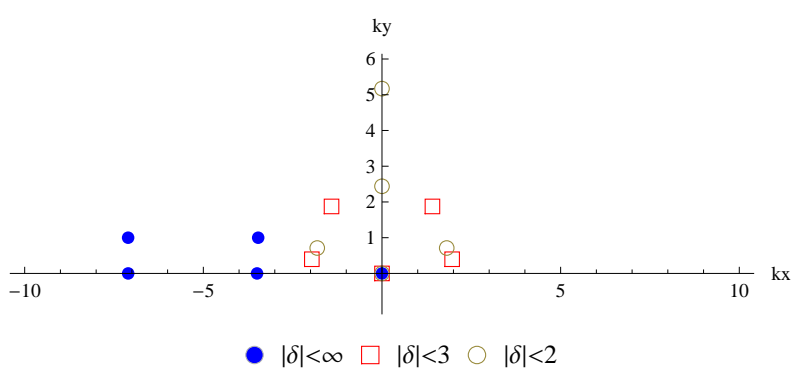

Fig. 10. Diagram of optimal arrays for broad-banded range of incident wave angle $\beta \in\left[\frac{\pi}{4}, \frac{3 \pi}{4}\right]$

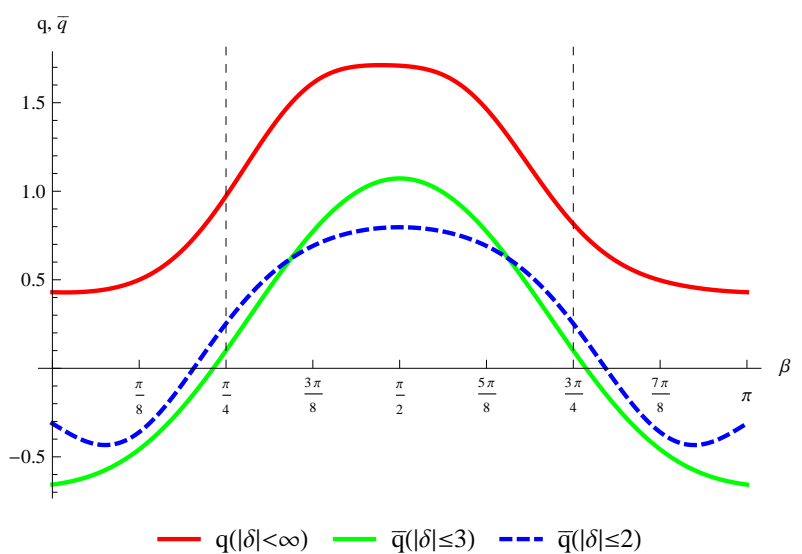

Fig. 11. Interaction factor against incident wave angle $\beta$ for optimal constrained and unconstrained five-WEC arrays, optimised for $\beta \in\left[\frac{\pi}{4}, \frac{3 \pi}{4}\right]$. The target range of wave angles is indicated by the vertical dashed lines.

factor values in Fig. 7. As with the narrow-banded regime, the largest motions are seen for the paired WECs. All five WECs exhibit $\delta>4$ and so violate the $\mathcal{O}(1)$ requirement of the model.

\section{Broad-Banded $\beta \in\left[\frac{\pi}{4}, \frac{3 \pi}{4}\right]$}

The optimal broad-banded array layouts for constrained and unconstrained motions are shown in Fig. 10. The unconstrained array layout is similar to the intermediate-banded case, with two closely spaced pairs of WECs separated to the right of the origin. Each pair had a separation of approximately 3.5 units between them and from WEC 1. Both the constrained arrays have a symmetric layout about the $y$-axis. In the $\delta_{\max }=3$ case, this is comprised by two WECs on each side of the $y$-axis. However, in the $\delta_{\max }=2$ case, there is one WEC on each side of the $y$-axis, while 2 WECs are placed along the $y$-axis and separated by approximately 2.5 units.

The behaviour of the optimal broad-banded arrays with $\beta$ variation is shown in Fig. 11. As with previous cases, the peak in performance occurs at or near the centre of the range of interest $\beta \in\left[\frac{\pi}{4}, \frac{3 \pi}{4}\right]$. Even in the unconstrained case, constructive interference is not maintained over the entirety of this range, since $q<1$ at the edges of $\beta \in\left[\frac{\pi}{4}, \frac{3 \pi}{4}\right]$. As expected, the constrained cases have a much reduced performance in comparison to the unconstrained case. The upper constraint of $\delta_{\max }=3$ achieves $q>1$ for a relatively

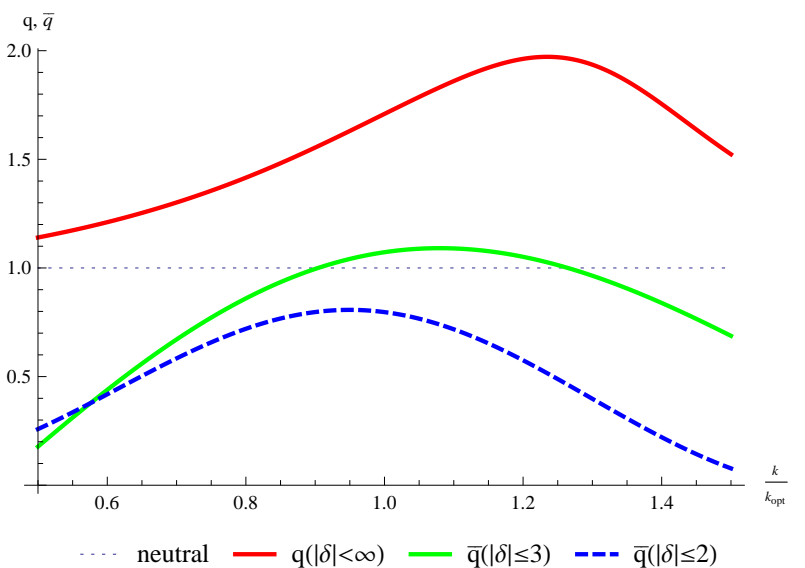

Fig. 12. Interaction factor against wavenumber $\frac{k}{k_{o p t}}$ for optimal constrained and unconstrained five-WEC arrays, optimised for $\beta \in\left[\frac{\pi}{4}, \frac{3 \pi}{4}\right]$, with $\beta=\frac{\pi}{2}$.

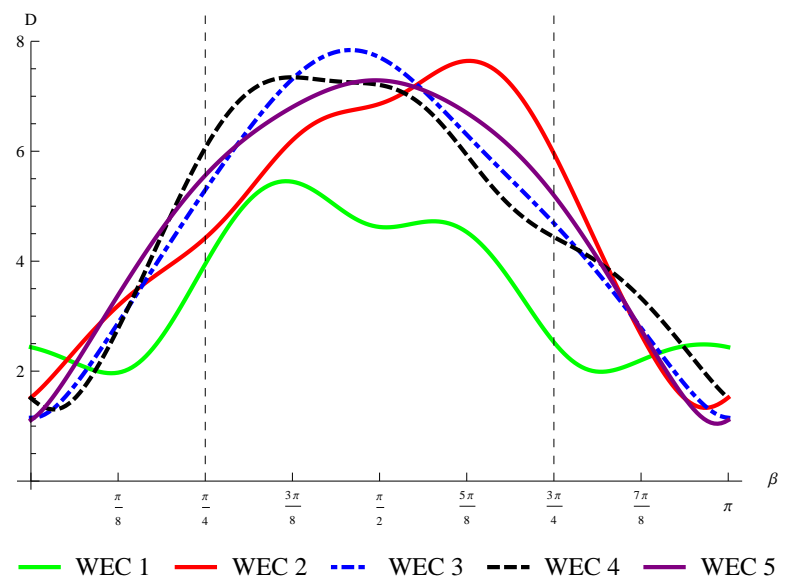

Fig. 13. Optimal displacement amplitudes against incident wave angle for optimal unconstrained five-WEC array, optimised for $\beta \in\left[\frac{\pi}{4}, \frac{3 \pi}{4}\right]$ (shown by vertical dashed lines)

small range of $\beta \in\left[\frac{7 \pi}{16}, \frac{9 \pi}{16}\right]$, with quite poor performance seen outside of this range. The $\delta_{\max }=2$ performance varies slightly slower but destructive interference dominates with $q<1$ for the entire range of interest.

The effect of variation of the wavenumber $\frac{k}{k_{o p t}}$ on the optimal broad-banded array performance is shown in Fig. 12. Once more, the unconstrained array maintains a $q>1$ performance for a $50 \%$ change in wavenumber but considerably lower performance is seen for the constrained arrays. The $\delta_{\text {max }}=3$ array achieves $q>1$ for $\frac{k}{k_{\text {opt }}} \in[0.9,1.26]$, whereas the $\delta_{\max }=2$ array has $q \leq 0.8$ for all values considered. As in the narrow and intermediate-banded case, the peaks in Fig. 12 occur slightly away from $\frac{k}{k_{o p t}}=1$ for all three arrays. The unconstrained array performance peaks at $\frac{k}{k_{o p t}} \approx 1.25$, while the $\delta_{\text {max }}=3$ array performance peaks at $\frac{k}{k_{o p t}} \approx 1.08$. Interestingly, the $\delta_{\max }=2$ array peaks at $\frac{k}{k_{o p t}} \approx 0.95$; this is the only case where the peak in performance with respect to wavenumber occurs at a value less than unity.

The optimal WEC displacement amplitudes for the unconstrained broad-banded array are presented in Fig. 13. As with 
narrow and intermediate-banded arrays, the motions of the paired devices (WECs 2 - 5) are larger than those for the isolated device (WEC 1). However, as with previous cases, all WECs maintain $\delta>4$ for the majority the range of interest $\beta \in\left[\frac{\pi}{4}, \frac{3 \pi}{4}\right]$. These WEC motions violate the $\mathcal{O}(1)$ requirement and so would invalidate the underlying linear wave theory.

\section{Discussion of Results}

This paper extends previous studies by considering the $\beta$ optimisation of general five-WEC arrays, without any prescribed geometry. This allows for a robust general layout optimisation and is the first time non-prescribed geometries are considered in this context. One difficulty with this increased generality is the associated increase in computational resources required for the optimisation, which limits the scope of investigations of this type. Utilising other optimisation routines, such as Genetic Algorithms, may decrease the run-time of the optimisation. However, this would also yield less knowledge of the evolution and reliability of the results due to the "blackbox" nature of such algorithms.

The optimal objective function values obtained in this paper are in general greater than the analogous results for linear arrays in [10], [12]. Better performing arrays are determined due to the increased freedom within the optimisation, as the WECs are not forced to lie on a straight line. It is also worth noting that although the WEC displacements of the unconstrained optimal arrays presented in this paper violate the $\mathcal{O}(1)$ requirement, they are two orders of magnitude smaller than the optimal linear array displacements from [10], [11].

It is clear that the optimal layouts obtained by the optimisation differ considerably depending on the range of wave angles permitted and the applied motion constraint. The unconstrained array layouts were all similar, with two pairs of closely-spaced WECs placed to one side of the $y$-axis. The optimal unconstrained narrow-banded array was placed to the right of the $y$-axis, while the analogous intermediate and broad-banded arrays were placed to the left. From considerations of symmetry, arrays mirrored about the $y$-axis perform the same and it is just a numerical artefact that the narrowbanded array resulted in WECs to the right instead of the left. It should also be noted that the WEC pairs are situated closer to the origin for broader $\beta$-ranges. In either case, the optimal unconstrained array is quite similar to two rows of linear WECs; it would be interesting to see what the optimal layout would be if say ten WECs were considered. However, this is numerically intractable due to the parameter explosion of including more WECs in the array.

The range of incident wave angle had a large impact on the optimal constrained array layouts, with noticeably different arrangements discovered in the constrained cases. For intermediate and broad-banded $\beta$-variation, the optimal constrained arrays were symmetric about the $y$-axis. This may seem expected, since the wave angle range considered is centred on $\beta=\frac{\pi}{2}$ and is also symmetric about the $y$ axis. This is in direct contrast to the optimal unconstrained layouts, which were placed to one side of the $y$-axis. In the narrow-banded case, asymmetric layouts were obtained as the best cases and the array found for each constraint were mirror reflections of each other about the $y$-axis.

Lower overall array performance was seen for the broader $\beta$-ranges, for both constrained and unconstrained motions. This is to be expected, since there are limitations on how well an array can perform constructively. For example, it is well known for unconstrained motions that the mean interaction factor over all wave angles is unity, as shown by [9]. A similar limitation would apply to constrained motions whereby the array cannot be forced to perform exceptionally well for a broad range of wave angles. Good performance can be achieved and maintained if a relatively small $\beta$-range is considered, as shown for the narrow-banded constrained arrays.

The trade-off identified previously in [12] between optimal performance and stability of performance is clearly shown in this paper for $\beta$-variation. If a broad-banded range of incident wave angle is the range of optimisation, this essentially requires that the array perform as well as possible over the entirety of this range of $\beta$. It is seen that broader ranges of $\beta$ produce lower objective function values and hence lower overall performance. However, another trade-off for linear arrays identified in [12] between $\beta$-stability and $k$-stability is not evidently observed in the results of this work. The $\frac{k}{k_{o p t}}$ curves are relatively stable and broadly similar for all ranges of $\beta$ considered. This suggests that this phenomenon was connected primarily with linear arrays and that optimising in a general two dimensional regime ameliorates this pitfall.

The best array performance is seen for the narrow-banded optimisation, where the range of interest is $\beta \in\left[\frac{7 \pi}{16}, \frac{9 \pi}{16}\right]$. Even for the $\delta_{\max }=3$ constraint, constructive interference was maintained throughout this range. Performance was also relatively stable to changes in wavenumber, with $\bar{q}>1$ being maintained for up to a $25 \%$ change in $k$. Thus, if a site is identified with a relatively low variation in both $\beta$ and $k$, which remain within the above limits, then this array may be an ideal choice.

In a preliminary study, the optimisation was tested at other wave angle ranges, before those targeting $\beta=\frac{\pi}{2}$ were chosen. In some cases, it was noted that a slightly better or a poorer optimal result was obtained at other wave angles, such as those centred on $\beta=0$. This improvement or deterioration was less than $1 \%$. In principle, the optimisation should be able to find the same array in both cases, due to symmetry. The optimal array at one angle should just be the rotation of the optimal array at a different angle. However, the same array may not be able to be found at other rotated angles due to the limits imposed on the variables. A rotation of $\frac{\pi}{2}$ may cause one of the WECs to be placed outside $k d_{j} \in[1,20] \cup \alpha_{j} \in$ $[0, \pi]$. Another possible explanation for this discrepancy is the large number of variables and the large size of the parameter space, particularly for the constrained optimisations. It may be that the optimisation failed to converge to the same results at different wave angles due the numerical intensity of the calculations, or some numerical difficulty at certain values of $\beta$. These problems cannot be avoided without a significant increase in the variable space within the optimisation. This 
would not only increase the space over which the optimisation must converge but also increase the starting point space for the WEC position variables, which would significantly reduce the performance of the optimisation routine. Thus the optimal arrays in this paper are not advertised as globally optimal, but are the best cases found within the computational limits present.

In some cases, the peak in $q$ against $\frac{k}{k_{o p t}}$ occurred at a value other than $\frac{k}{k_{o p t}}=1$. This suggests that an increased performance could be achieved by having the non-dimensional radial positions of the WECs $\left(k d_{j}\right)$ slightly larger or smaller. However, given that the optimisation routine did not converge to such a case, this suggests that the increased performance at the larger/smaller $k d_{j}$ values also gives an increased $\beta$ sensitivity and poorer performance over the range of interest of $\beta$, as this was the main consideration within the objective function. It also points towards the possible trade-off between stability in some array variables and sensitivity to others. However, the effect is very slight, as the peaks in $\frac{k}{k_{o p t}}$ occurred very close to unity in all cases.

In many physical array sites, the variation of incident wave angle will not be uniform about a given range. In most cases, there is a spectral behaviour where most of the wave would come from a range of angles, with decreased resource from angles outside this range. To assess the effect of this concentration of the resource, an alteration of the objective function is proposed, where a weighting function is included in the integral. The weighting function must be normalised to maintain consistency. This type of optimisation would give a more detailed idea of the effect of $\beta$-variation, as spectrum variability is incorporated in this implementation in some sense. Since regular waves and ideal power absorption are still assumed in the unconstrained optimisation, this objective function would essentially assume that ideal power is absorbed at all wave angles.

\section{ACKNOWLEDGMENT}

$\mathrm{PhD}$ funding for Justin McGuinness was provided by a Government of Ireland Postgraduate Scholarship from the Irish Research Council and is gratefully acknowledged.

\section{REFERENCES}

[1] D. V. Evans, 1979, "Some theoretical aspects of three-dimensional wave-energy absorbers," Proc. First Symposium on Ocean Wave Energy Utilization, Gothenburg, Sweden.

[2] J. Falnes, 1980, "Radiation impedance matrix and optimum power absorption for interacting oscillators in surface waves," Appl Ocean Res, vol. 2, no. 2, pp. 75-80.

[3] G. P. Thomas \& D. V. Evans, 1981, "Arrays of three-dimensional waveenergy absorbers," J Fluid Mech, vol. 108, pp. 67-88.

[4] P. McIver, 1994, "Some hydrodynamic aspects of arrays of wave-energy devices," Appl Ocean Res, vol. 16, no. 2, pp. 61-69.

[5] S. A. Mavrakos \& P. McIver, 1997, "Comparison of methods for computing hydrodynamic characteristics of arrays of wave power devices," Appl Ocean Res, vol. 19, no. 5-6, pp. 283-291.

[6] C. Fitzgerald \& G. P. Thomas, 2007, "A preliminary study on the optimal formation of an array of wave power devices," Proc. Seventh European Wave and Tidal Energy Conferance, Porto, Portugal.

[7] B. F. M. Child, 2011, "On the configuration of arrays of floating wave energy converters," PhD thesis, The University of Edinburgh.

[8] J. P. L. McGuinness, 2018, "Hydrodynamic optimisation of an array of wave-power devices," $\mathrm{PhD}$ thesis, University College Cork.

[9] C. Fitzgerald, 2006, "Optimal configurations of arrays of wave power devices," Master's thesis, University College Cork.

[10] J. P. L. McGuinness \& G. P. Thomas, 2015, "Optimal arrangements of elementary arrays of wave-power devices," Proc. Eleventh European Wave and Tidal Energy Conference, Nantes, France, 10 pages.

[11] J. P. L. McGuinness \& G. P. Thomas, 2016, "Hydrodynamic optimisation of small arrays of heaving point absorbers," Journal of Ocean Engineering and Marine Energy, vol. 2, no. 4, pp. 439-457.

[12] J. P. L. McGuinness \& G. P. Thomas, 2017, "The constrained optimisation of small linear arrays of heaving point absorbers. part i: The influence of spacing," International Journal of Marine Energy, vol. 20, pp. $33-44$.

[13] J. P. L. McGuinness \& G. P. Thomas, 2017, "Optimisation of elementary arrays of wave-power devices in irregular waves," Proc. 12th European Wave and Tidal Energy Conference, Cork, Ireland, 10 pages.

[14] T. Havelock, 1955, "Waves due to a floating sphere making periodic heaving oscillations," P Roy Soc A-Math Phy, vol. 231, no. 1184, pp. $1-7$.

[15] P. E. Gill, W. Murray, M. A. Saunders, \& M. H. Wright, "User's guide for npsol (version 4.0): A fortran package for nonlinear programming." DTIC Document, Tech. Rep., 1986. 